\title{
IL DATIVO ETICO NEL FRIULANO SONZIACO
}

1. Nei suoi ricordi giovanili, scritti nel secondo dopoguerra e pubblicati nella rivista friulana Sot la Nape negli anni settanta e ottanta del secolo scorso, riuniti poi nel libro Di cà e di là da la Grapa. Di cà e di là dal Pomeri. Blecs gurizans Luciano Spangher, goriziano, benemerito cultore della cultura e tradizioni della sua città, offre una abbondante quantità di esempi in cui si trova un uso particolare del pronome personale atono, al dativo, sensibilmente indebolito al livello semantico, quel fenomeno, dunque, che le grammatiche del latino sogliono classificare come dativus ethicus. Pensiamo ai passi come il seguente in cui Spangher descrive il ghetto goriziano e la pace che vi regnava tra gli appartenenti alle varie religioni della città: In ta ciasis stavin cristians, obreos e ancia protestanz e duc' ti vivevin di amor e di acordo, p. 47. Il pronome personale, evidentemente, non ha un pieno valore semantico.

2. L'indebolimento del significato di un elemento lessicale è un fenomeno universale. Nella transizione dal latino verso le lingue romanze sarà sufficiente ricordare gli attuali valori dei sostantivi come CAUSA, RES, anche MENS, -TIS il cui ablativo -mente è un mero espediente grammaticale, morfema per la formazione dell'avverbio di modo. Inoltre, le funzioni dei verbi, come ESSE o HABERE o VELLE/* volere che dai verbi di pieno significato sono diventati espedienti per la formazione dei paradigmi verbali analitici, oppure ausiliari, modali, fattitivi; e poi, l'indebolimento semantico è palese nelle sorti del numerale UNUS, UNA che attraverso il valore di pronome indefinito arriva alla funzione dell'articolo indeterminativo.

Si può constatare l'indebolimento semantico anche nell'uso delle forme atone al nominativo del pronome personale nelle lingue che esigono l'impiego del pronome personale accanto alle forme verbali, come in francese con je parle 0 , molto più rigorosamente, in friulano con jo 'o feveli o Sef al fevele. Però, all'infuori dell'uso con le forme verbali, i pronomi personali, nelle forme toniche e atone, appunto perchè pro-nomi, conservano il valore semantico del sostantivo che sostituiscono per ragioni di stile, nella ricerca di alleggerire la frase, soprattutto. Il pronome atono conserva il significato del sostantivo che è chiamato a rimpiazzare. Nella sequenza di proposizioni come Ho comprato il/un libro e lo sto leggendo il pensiero del parlante è chiaramente espresso, giacchè il pronome personale atono nella seconda proposizione rende pienamente il significato del sostantivo nella prima. Non lo è, invece, nella costruzione, in apparenza addirittura più chiara, però insolita e per di più goffa e maldestra, con la ripetizione dell'oggetto diretto: Ho comprato il/un libro e sto leggendo il/un libro.

3. Non così in un uso specifico del pronome personale, in forma atona, dalle grammatiche del latino chiamato DATIVUS ETHICUS che alcune accostano al 
DATIVUS (IN)COMMODI. La grande Lateinische Grammatik, Szantyr 1965, p. 93, lo tratta nel capitolo di quest'ultimo; ammonisce però nello stesso tempo che la nomenclatura è discutibile, a volte fuorviante. Ad ogni modo, asserisce Szantyr, il dativo etico fa parte del linguaggio colloquiale e il suo uso può sembrare addirittura superfluo, stando a uno stile asciutto e ponderato. L'impiego del pronome, secondo Szantyr, cerca di suscitare l'interesse dell'interlocutore: $» E$ Er tritt vor allem in der Erzählung auf und soll hier lediglich die Aufmerksamkeit oder das Interesse des Hörers an der Satzaussage (änlich wie z. B. eingeschobenes tibi dico) erwecken und festhalten«.

Le grammatiche del latino mettono inoltre in rilievo il fatto che la persona cui va attribuito l'interesse $o$, meglio, un sentimento non è necessariamente la stessa a quella del soggetto dell'azione di cui parla la proposizione; illustrano il fenomeno con degli esempi come: Quid mihi Celsus agit?, Quid tibi vis?, Ecce tibi!

4. Nelle lingue romanze tale uso del pronome nel dativo si è conservato, benchè in alcune di loro non sia molto frequente. Per l'italiano, Rohlfs III, par. 640, accenna alla notevole diffusione dell'uso di questo dativo (i traduttori hanno scelto come termine adatto quello di «sentimento di animo personale») e menziona esempi letterari, come Il padre te la fece chiudere in una torre (Grossi), mentre per l'Italia meridionale constata la frequenza del dativo nei verbi di interesse personale (mangiarsi, sic. pòrtatilu, ecc.) i quali, per dir il vero, chiamano in causa piuttosto l'interesse, il «pro» della persona che parla e di conseguenza non entrano strettamente nell'ambito del dativo etico, benchè gli siano, per il senso, molto vicini.

Giacchè, per poter vedere senz'ombra di dubbio in un complemento indiretto il dativo etico, l'interesse deve essere escluso. Una semplice frase come ti ho mangiato una mela si presta a due interpretazioni diverse e, per chi ascolta, dobbiamo supporre un'esperienza extralinguistica, vale a dire la conoscenza della situazione reale. Troviamo un esempio convincente nella Grande grammatica italiana di consultazione, Renzi 1988, I, p. 66, dove viene messa in rilievo la distinzione tra il dativo etico e il benefattivo (= d'interesse): Ed ecco che Maria ti stira le camicie di Pietro senza pretendere un compenso. Se il complemento indiretto ti è sostituibile con un sintagma come per te, abbiamo a che fare con il benefattivo; se invece è interpretabile come dativo etico, spiega giustamente Renzi, il suo significato è parafrasabile con Ed ecco Maria che stira le camicie di Pietro ... e di questo dovresti meravigliarti, o qualcosa di simile.

Con tutto, pare che l'impiego del dativo etico in italiano, nei testi scritti, stando anche al poco spazio che le grammatiche gli riservano, non sia molto frequente; ripetiamo che tale uso è da aspettarsi, semmai, in uno stile colloquiale.

5. Non è abituale, così pare, nemmeno in friulano, benchè non sia proprio sconosciuto. Lo registra Faggin 1997, p. 102. Nota che in friulano tale uso esiste, «che a rigore è superfluo, ma che in realtà può dare vivacità e calore a un discorso» e aggiunge due esempi, di cui l'uno è di carattere letterario: viôd di pašiju ben, che no mi si sclagnišin 'cerca di nutrirli bene, chè non smagriscano (che non mi si smagriscano)' e un altro, più poetico, un garbinut sutil ti dispičhave / dai morârs sul prât sin la ultime fuee 'un lieve garbino staccava dagli alberi sul prato, una dopo l'altra, 
tutte le foglie'. Gli altri trattamenti del pronome personale nelle grammatiche del friulano non ne fanno menzione, e nemmeno il Lexikon der romanistischen Linguistik, vol. III; si potrebbe dire di conseguenza che almeno il friulano contemporaneo non ne faccia un uso abbondante.

6. Diversa è la situazione nel territorio che ci interessa più da vicino e, più ampiamente, quella dove vengono a contatto le parlate slave e quelle romanze; di queste ultime, il friulano e il veneto. Il fenomeno non è rimasto inosservato. Una prima menzione la fece già Schuchardt nel suo omaggio a Miklošič, Slawo-deutsches und slawo-italienisches del 1884, in cui (pp. 109-110), volgendo lo sguardo soprattutto all'influsso del ceco sul tedesco, accennava però anche alle interferenze tra il croato e il veneto di Dalmazia e citava addirittura un passo del religioso cinquecentesco sloveno Primož Trubar, esponente più alto della Riforma protestante in Slovenia e con le sue prime traduzioni bibliche in sloveno il fondatore della lingua scritta, che è Od kod ste si vi? 'e voi, da dove siete?' col pronome riflessivo atono, al dativo, a rigore superfluo. Menzionava inoltre Schuchardt per Zara Come ti me sta? (kako mi ti?), frase propria, diceva, anche ai cittadini colti: vediamo, qui, il dativo etico nel dativo del pronome personale della 1.a persona, me croato mi. Riporta, poi, un bell'esempio, sempre da Zara, cosa ti fa signora? (kako ti je gospoja?) e un altro da Spalato ghe xe amalada la madre e nota il legame semantico del dativo etico e la nozione del possessivo, visibile soprattutto nel secondo esempio 'gli si è ammalata la madre', 'ha ammalata la madre'. Schuchardt ha trovato un uso simile nell'interferenza dello ceco sul tedesco: cita mein Suhn is-e s i c h Professer e lo giudica analogo a mi se xe de Pisin, annotando «eine slawische Färbung».

Cinquant'anni più tardi tornò sulla questione Carlo Tagliavini nel suo esauriente studio Sugli elementi italiani del croato in Italia e Croazia, Roma 1942-XX, pp. 371-454. E' vero che la pubblicazione andava in una direzione soltanto: era destinata più che altro a illustrare le varie influenze della civiltà italiana (lingua, letteratura, arti) sul croato della costa dalmata. Tagliavini, a pag. 381, tuttavia, seppe mettere in rilievo anche l'influsso linguistico delle parlate slave, croate, sul veneto della costa adriatica orientale e annotò l'uso frequente delle frasi come Come ti me sta? ma ti me xe de mal (a Fiume, ad Arbe, a Zara).

L'impiego del dativo etico è inoltre molto frequente nella lingua delle storielle che parlano dell'ambiente istro-quarnerino, create dagli autori Carpinteri e Faraguna. La lingua di queste storie è scorrevole, sembra molto naturale, esposta agli influssi linguistici croati, a cominciare dal titolo Le Maldobrie, e soprattutto, per quel che ci riguarda, sorta da una vena facile, colloquiale, o meglio, perchè si tratta di un colloquio un po' finto, di un racconto immediato. Basterà copiare qualche esempio da uno dei loro lavori sotto il titolo "L'Austria era un paese ordinato": Insoma, ti te bevi che te bevi, ierimo mborezadi; Bisogna che vù dimani bonora me andè in Amburgo; Franz Fèrdinand l'Arciduca Francesco Ferdinando, quel che ve xe deventà Erede dela Duplice, rispett. pp. 113, 273. Oltre che per mostrare la frequenza dell'impiego del dativo in questa funzione, i passi sono interessanti perchè il dativo semanticamente affievolito non è solo quello della 2.a sg. 
7. Per limitarci ora al friulano sonziaco, ripetiamo che il nostro interesse è stato suscitato dalla lingua dello Spangher. L'impiego del dativo etico è abbondante, forse addirittura sovrabbondante e, sia menzionato subito, il pronome personale appare sempre alla 2.a persona del singolare. Nei suoi ricordi, nel capitolo Il Ghet, lo scrittore goriziano, nato nel 1923, fa un panorama storico della comunità ebraica goriziana e aggiunge memorie personali riguardo ai suoi coetanei e compagni di classe ebrei nel decennio prima dello scoppio della seconda guerra mondiale. Su qualcosa come dieci pagine di testo, pp. 47-59, troviamo una quarantina di esempi col pronome personale atono al dativo con chiaro valore di dativo etico. Al passo citato all'introdurre il nostro modesto contributo aggiungeremo solo alcuni pochi: In becaria ti vignivin servì dal Kizlinger, che ti curava i bocòns che compravin i obreos; Dopo non ti mangiavin purzel e par chist fàt ti dopravin un grun di ocis; Tal 1800 la comunitàt ti si veva slargiàt cun gnovis migrazions che ti vignivin da l'Europa oriental; Il 23 di novembar dal 1943, podopo, chista ('la comunità ebraica') ti jà ciapat l'ultim colp. 45 obreos, di chêi che no ti si vevin scundùt /.../ son stas puartâs via dai todesc.

Ripetiamo: la lingua di Spangher è scorrevole, quasi volutamente frivola e può servire come conferma delle constatazioni di Szantyr sopra l'uso del dativo etico in latino. Abbiamo, infatti, nello stesso friulano goriziano, sonziaco, uno scrittore dove il dativo etico non si trova affatto. Pensiamo alla lingua di Mario Ranieri Cossàr, un altro benemerito ricercatore delle cose goriziane. Le sue Storiutis gurizzanis sono cupe, a volte terrificanti, non adatte, di certo, a esser lette al bambino perchè questi si addormentasse, benchè lo scrittore stesso dica d'averle sentite raccontare dalla madre. Sono tetre nel contenuto e nella lingua. Vogliamo dire, non hanno quella leggerezza, quella frivolezza scorrevole dello Spangher. Cossàr inizia tutte le storie con una frase come In t'un cias'cel dal Friul vivevin par antic $i$ conz dai Coss, p. 29; ai timps dal vescul Attimis vivevin a Gurizza doi fradis, p. 45, vale a dire, senza ricorrere all'uso del dativo etico. Va aggiunto che i Dialoghi piacevoli in dialetto vernacolo triestino di Giuseppe Mainati, che pur offrono l'immagine linguistica del tergestino, vale a dire, del friulano meridionale settecentesco, e per di più sotto forma di conversazione o di pacata narrazione, non hanno esempi convincenti dell'uso di tale forma del pronome personale con valore di dativo etico. Hanno tuttavia esempi che fanno pensare a quelli latini. Nel Dialogo terzo leggiamo Se met im fond del arnàs de òi diès per zent de mèi, e se ti uosto, ànchia dei per, o zariesis mastruzàdis che Mainati stesso ha tradotto come 'Si mette in fondo ai vasi dell'olio un dieci per cento di pomi, o pere, o cerase infrante': nella traduzione ha omesso, dunque, l'inserto con il verosimile dativo etico.

Non sorprende che il pronome personale con tale valore non appaia nelle traduzioni, giacchè, secondo noi, il traduttore opera in modo molto più ligio alla norma lettteraria scritta, che non il vero creatore cui l'estro suggerisce a volte variazioni di stile, ricorsi al parlato, impiego delle forme o strutture insolite.

8. Ci domandiamo se il ricorso al dativo etico in Spangher sia da considerare un tratto personale. Vorremmo mettere in rilievo il parere di molti linguisti che si sono occupati di questo uso stilistico sullo stretto legame semantico del dativo etico con 
la nozione di appartenenza, di possesso, affievolito e non completamente materiale. Schuchardt aveva notato questo fenomeno nella parlata tedesca, influenzata dal ceco, e visto nel veneto parlato sulla costa dalmata, come nell'esempio citato: cosa $t i$ fa signora?

E' noto che il dativo, nel romanzo orientale almeno, a volte serve a coniare strutture con più o meno vaga sfumatura di possesso. Un lontano precedente si trova nel latino con LIBER MIHI EST, l'impiego che in qualche modo continua fino ai giorni nostri, ad es. in romeno in mi-e sete, mentre le lingue romanze centrali e occidentali ricorrono ai costrutti di espressione più concreta con i verbi HABERE e TENERE, dove non può esserci necessità del pronome personale al dativo. La nozione di possesso, di appartenenza, fortemente mitigata, conserva in romeno il costrutto col dativo del pronome personale: tata lui 'suo padre'. La nozione di proprietà è nel romanzo normalmente resa per il mezzo del genitivo, mentre il latino orientale, e poi il romeno, non ha difficoltà di usare il dativo: casa vecinului 'la casa del vicino', columna lui Traian 'colonna di Traiano', stelele cerului 'le stelle del cielo' sono esempi che dà Bourciez, 496 a, e ammette come possibile l'influsso slavo, «car en slave, spiega, le datif adnominal était largement développé». Infatti, l'influenza dello slavo sul romeno, immerso nel mondo slavo, è stata fortissima nell'arco di molti secoli, dai primi contatti ancora nella tarda antichità fino ai giorni nostri. Effettivamente, l'ambiente slavo conosce un ampio uso del pronome personale al dativo, in un certo qual senso legato all'idea di un vago, mitigato possesso. Alla mia domanda posta anni fa a uno studente serbo sul perchè studiasse a Ljubljana ebbi la risposta tu mi je brat ho qui il fratello', alla lettera 'qui mi sta il fratello'.

9. Tale uso sembra più spontaneo nelle lingue slave sud-orientali che non in sloveno. Se proponiamo come spiegazione per l'uso del dativo etico nel friulano goriziano l'influsso sloveno, vale a dire un calco sintattico-semantico secondo il modulo conosciuto allo sloveno, ci basiamo sul fatto che nel friulano centrale e nei dialetti veneti tale uso non è molto noto; nel Goldoni, ad esempio, si trovano pochi passi col dativo. Per contro, lo sloveno lo conosce: se ne scoprono esempi abbondanti nella letteratura e non mancano neanche nel parlato odierno. La grammatica per lo sloveno di Jože Toporišič, Slovenska slovnica, 4.a ed., del 2004, p. 270, offre passi come Da si mi zdrav 'che tu mi sia sano' e li giudica come stato d'animo personale. Per lo Spangher, poi, possiamo aggiungere che sua nonna paterna era una slovena di Cromberg. Inoltre, egli stesso si rammarica del fatto di non aver imparato lo sloveno come avrebbe potuto, spiegandone anche la vera ragione proprio all'inizio dei suoi ricordi: Mi dispiàs dome, di chêl timp, di no vè imparàt a ciacarà par sclàf, come che varès podût, ma saveso simût che jara: ogni sàbida dovevi là dai «balila», e là nus disevin che si podeva fevelà dome par talian. Stupidadis! Comunque è più che probabile che, vivendo a Gorizia, abbia avuto contatti diretti con la lingua slovena: ne vediamo una conferma anche nel fatto che nei suoi ricordi giovanili, vale a dire a distanza di quarant'anni, ricorda ancora i suoi compagni di classe sloveni e i loro cognomi, italianizzati, a volte anche quelli originali: Vogric, Gomiscek, Cocianni, Sciurek, Policek, Slamich, Collini/Collenz, Vescovo/Skoff, Wili Papes. 
Bibliografia

BouRCIEZ (1967): Bourciez, E., Eléments de linguistique romane, Paris, Klienksieck

CARPINTERI \& Faraguna (1996): Carpinteri, L. e Faraguna, M., Le Maldobrie, LAustria era un paese ordinato, 2.a ed., Trieste, MGS Press Editrice.

Cossar (1930): Cossàr, R. M., Storiutis Gurizzanis, Udin, 1930 - VIII, Società Filològiche furlane "G.I. Ascoli".

DLALOGHI (1972): I dialoghi piacevoli in dialetto vernacolo triestino. Pubblicati e tradotti da Giuseppe Mainati. Edizione critica a cura di Mario Doria, Trieste, Edizioni "Italo Svevo".

FAGGnN (1997): Faggin G., Grammatica friulana, Udine 1997, Ribis.

RENZI 1988: Grande grammatica italiana di consultazione a cura di Lorenzo Renzi e Giampaolo Salvi, vol. II. Bologna 1988, Il Mulino.

ROHLFS III (1969): Rohlfs, G., Grammatica storica della lingua italiana e dei suoi dialetti. Sintassi e formazione delle parole, Torino 1969, Giulio Einaudi Editore.

SchuchaRDT (1884); Schuchardt, H., Slawo-deutsches und slawo-italienisches, Graz 1884, Leuschner \& Lubensky.

SPANGHeR (1990): Spangher, L., Di cà e đi là da la Grapa. Di cà e di là dal Pomeri. Blecs gurizans, Guriza 1990, Società Filologica Friulana.

SZANTYR (1965): Leumann-Hofmann-Szantyr, Lateinische Syntax und Stilistik, München, 1965, C.H. Beck'sche Verlagsbuchhandlung.

TAGLIAVINI (1942): Tagliavini, C., Sugli elementi italiani del croato in Italia e Croazia, Roma 1942-XX, Accademia Reale d'Italia.

TOPORIŠIČ (2004): Toporišič, J., Slovenska slovnica, 4. ed., Maribor, 2004, Obzorja.

\section{Povzetek \\ ETIČNI DATIV V OBSOŠKI FURLANŠČINI}

$\mathrm{V}$ jeziku ugotavljamo pogostno slabljenje pomena jezikovnih prvin. Za romanski svet, za prehod iz latinščine v romanske jezike, je mogoče najti obilo primerov: lat. samostalnik MENS, -TIS, je v svoji sklonski ablativni obliki postal morfem za tvorbo prislovov načina, polnopomenski glagoli kot ESSE, HABERE, VELLE (ljudsko *volere) so največkrat pomožni glagoli in so skladenjske prvine sestavljenih glagolskih oblik, števnik UNUS, UNA je postal prek vrednosti nedoločnega zaimka nedoločni člen.

Ne takó osebni zaimek, razen tam, kjer je sestavna prvina glagolskih paradigem, npr. v francoščini in tudi $\vee$ furlanščini. Sicer pa ohranja osebni zaimek polno pomensko vrednost. $V$ vseh jezikih je nepogrešljivo jezikovno sredstvo, da se govoreči izogne ponavljanju kakega samostalnika: raba nenaglašenega zaimka zmeraj docela jasno izraža njegovo misel.

$\mathrm{Ni}$ pa ohranjen polni pomen $\mathrm{v}$ neki posebni rabi sklonske oblike osebnega zaimka $\mathrm{v}$ dajalniku in tedaj ga slovnice latinskega jezika imenujejo dativus ethicus. V tej rabi je pomen zaimka docela oslabel: sklonska oblika $\mathrm{v}$ dajalniku se ne sklada $\mathrm{z}$ osebkom stavka. Raba se zdi odvečna in nepotrebna, če je v jeziku sploh mogoče govoriti o odvečnosti. Za suho, strogo urejeno izjavo je to morda res, a stilistična vrednost etičnega dativa je ravno $v$ tem, da pritegne pozornost sogovornika. Etični dativ je bil znan v latinščini in je znan, čeprav ne preveč rabljen, v sodobnih romanskih jezikih in bi potemtakem rabo lahko imeli za dediščino iz latinščine. Vendar so izraziti primeri in pogostnost rabe dajalnika na stičnih romansko-slovanskih ozemljih (Trst, Istra, v preteklosti tudi Dalmacija) vzbudili zanimanje jezikoslovcev, že od Schuchardta (1884) dalje. Za obsoško, goriško furlanščino mislimo, da gre za vpliv slovenskih zahodnih govorov, ki na furlanščino mejijo že stoletja, oziroma $z$ njo ozemlje delijo. Pojav je najbrž v slovenščini manj znan kot $v$ hrvaščini ali srbščini, a Toporišič, Slovenska slovnica, 4. izd., 2004, ta skladenjski pojav pri zaimku ugotavlja, opozarja v tej rabi na čustveno zavzetost in na dejstvo, da se dajalniška oblika zaimka pojavlja v 1. in 2. os. ednine in množine. Navaja primere iz slovenske književnosti in tudi iz pogovornega jezika: To vam je bilo živo na ribniku! Ponekod viđi povezanost med čustvenostjo in svojilnostjo: Kje tihi si mi dom, ti sreča moja prava. - Naj končamo z verzom iz slovenskega epskega pesništva: Pa bil je Martin svoje dni vojak vam, na straži stal. 